\title{
Combining Ability for Winter Survival and Turf Quality Traits in St. Augustinegrass
}

\author{
Jennifer A. Kimball and Thomas G. Isleib \\ Department of Crop Science, North Carolina State University, Box 7620, \\ Raleigh, NC 27695-7620
}

William C. Reynolds

Department of Soil and Crop Sciences, 370 Olsen Boulevard, College Station, TX 77843

\section{Maria C. Zuleta and Susana R. Milla-Lewis ${ }^{1}$ \\ Department of Crop Science, North Carolina State University, Box 7620, Raleigh, NC 27695-7620}

Additional index words. st. augustinegrass, combining ability, diallel mating design, winter survival, turf quality

\begin{abstract}
New elite st. augustinegrass cultivars with improved cold tolerance and desirable turf quality are needed for the turf industry, especially in the transitional climatic region of the United States. To efficiently use sources of cold tolerance in a breeding program, an understanding of the genetic control of this trait and its relationship to important turf quality traits is required. Therefore, the objective of this study was to estimate general and specific combining abilities for cold response and turf quality traits. Six diploid genotypes of st. augustinegrass were selected as parents for a diallel mating design without reciprocals and evaluated over 3 years at two locations. The true hybridity of crosses was confirmed using simple sequence repeats (SSR). Combining ability analysis revealed that both general and specific combining abilities were significant across years and locations. Specific combining ability (SCA) was the largest source of genetic variation for winter survival, genetic color, turf density, and end-of-season cover indicating that nonadditive gene effects play a key role in the inheritance of these traits. The parental genotype 'GF2' was identified as a promising parent for future breeding efforts as it provided positive general combining ability (GCA) effects for both cold tolerance and turf quality traits, which were not significantly correlated with one another. Lines identified as parental selfs generally showed lower cold response and inferior turf quality than the original parental lines indicating that inbreeding depression can occur in st. augustinegrass. This study provides information regarding the combining ability of cold response and turf quality traits in st. augustinegrass, which will ultimately aid in parental selection for our future breeding efforts.
\end{abstract}

St. augustinegrass [Stenotaphrum secundatum (Walt.) Kuntz] is a warm-season, perennial grass species commonly used in the turfgrass industry for its superior shade tolerance and stoloniferous growth habit. However, winter survival is a major limiting factor for the species affecting its area of adaptation and marketability, especially in the transition zone of the United States. In addition, the most cold-tolerant cultivars

Received for publication 2 Mar. 2016. Accepted for publication 10 May 2016.

This research was supported in part by funding provided by the North Carolina Crop Improvement Association and the North Carolina State University Center for Turfgrass Environmental Research and Education.

We would like to thank the personnel at the Upper Mountain Research Station (Laurel Springs, NC) and the Turfgrass Field Lab (Raleigh, NC) for help maintaining research plots.

${ }^{1}$ Corresponding author. E-mail: susana_millalewis@ncsu.edu. characteristics, but similar to less cold hardiness (Reynolds et al., 2009). On the other hand, available commercial cultivars with good turf quality often have limited cold tolerance. 'Seville' (Riordan et al., 1980), a popular cultivar well liked for its semidwarf habit, fine-leaf texture, and dark green color, often exhibits high levels of winterkill (Moseley et al., 2010; Philley et al., 1996; Riordan et al., 1980).

Despite the identification of cold tolerant germplasm, limited progress has been made in breeding for improved winter survival in st. augustinegrass. This is in part due to the complexity of the trait, insufficient genetic knowledge of tolerance components, lack of efficient selection criteria, and limited breeding efforts for this trait. For example, 'Raleigh' is still considered an industry standard for cold hardiness in the species 30 years after its release. Previously, Philley et al. (1998) evaluated the inheritance of winter survival in st. augustinegrass with eight diploid genotypes using a diallel analysis. General combining ability was the largest source of variation for winter survival and lethal temperature. The large additive variance indicated that st. augustinegrass should respond to selection (Philley et al., 1998). However, field trials were conducted in Mississippi and may not reflect genotype performance in the upper transition zone. In addition, turf quality was not reported in the study and knowledge of the transferability of turf quality traits from parents to progeny in combination with winter survival in st. augustinegrass is important in the development of new, elite cultivars.

A set of 'Raleigh' variants selected for high levels of winter survival as well as improved turf quality (Reynolds et al., 2009) and collections from home lawns in Raleigh, NC, selected for early spring green-up were identified as potential parents for cold tolerant population development. To efficiently use these resources in our breeding program, the objective of this study was to estimate their general and specific combining ability for winter survival and turf quality traits. cusistics, such as finer leaf texture shorter internode length that are desirable in the market. Therefore, new st. augustinegrass cultivars with improved winter survival and desirable turf quality are needed for the turfgrass industry in the transitional climatic region of the United States.

Studies have reported significant variability in winter survival and freezing tolerance among present-day cultivars (Kimball et al., 2016a, 2016b; Milla-Lewis et al., 2013; Moseley et al., 2010; Reynolds et al., 2009). Cold hardy cultivar, Raleigh (Bateman, 1980), performs consistently well in the upper range of the species' geographic limits. However, 'Raleigh's' coarser leaf texture and longer internodes when compared with other st. augustinegrass cultivars as well as other warm-season turfgrasses are often undesirable (Reynolds et al., 2009). Somatic variants and gamma-ray mutants of 'Raleigh' were developed in 2004 (Li, 2007). In comparison with 'Raleigh', they had improved aesthetic

\section{Materials and Methods}

Six diploid $(2 n=2 x=18)$ genotypes of st. augustinegrass were selected as parents for a diallel mating design without reciprocals based on their high levels of winter survival and/or turf quality (Table 1). Reciprocals were not used in this study as Philley et al. (1998) found no maternal effects in their st. augustinegrass heritability study. The six genotypes included: cultivars Raleigh and Seville; experimental lines 'GF2', a gamma radiation-derived 'Raleigh' mutant, and 106T3, a somaclonal variant of 'Raleigh'; and accessions ' $\mathrm{C} 1$ ' and '1800S', ecotypes collected from old home lawns in Raleigh, $\mathrm{NC}$, selected for early spring green-up ( $\mathrm{Li}$, 2007). Crosses were made according to the protocol of Genovesi et al. (2009) during the fall of 2009. One to three SSR markers 
developed by Mulkey et al. (2013) were used to validate that products from crosses were true hybrids following the protocols outlined in that study. Parental selfs identified by SSR markers were included in this study (Table 2).

Three replications of each single cross, parental self, and the six parents were planted in a randomized complete block design in $1-\mathrm{m}^{2}$ plots at the North Carolina State University Lake Wheeler Turfgrass Field Laboratory in Raleigh, NC, and at the Upper Mountain Research Station in Laurel Springs, NC, in June 2011. Plots were mowed weekly at a height of $6.35 \mathrm{~cm}$, fertilized monthly at a rate of $48.8 \mathrm{~kg} \cdot \mathrm{ha}^{-1}$ from May to August to accumulate $195.2 \mathrm{~kg} \cdot \mathrm{ha}^{-1}$ for a year, and irrigated to avoid drought stress.

Traits were grouped into three categories: cold response including winter survival and spring green-up; turf quality traits, including overall turf quality, leaf texture, genetic color, and turf density; and establishment, including midseason and end-of-season cover. Traits were evaluated over a 3-year period from 2011 to 2014. Spring green-up ratings were taken in April and winter survival ratings were collected in May. Turf quality traits and midseason cover were rated in July, while end-of-season cover was rated in October of each year. Midseason and end-of-season cover of individual plots were rated on a $0 \%$ to $100 \%$ scale. The remaining traits were evaluated visually on a 1 to 9 scale according to the National Turfgrass Evaluation Program (NTEP, 2012) as follows:

Table 1. St. augustinegrass cultivars, ecotypes, and experimental genotypes used in the United States and their breeding information and release date.

\begin{tabular}{|c|c|c|c|c|c|}
\hline No. & Parent & Type & $\begin{array}{l}\text { Experimental } \\
\text { notation }\end{array}$ & Source/breeder & Yr released \\
\hline 1 & Raleigh & Cultivar & NA & NCSU, Raleigh, NC & $1983^{z}$ \\
\hline 2 & $1800 \mathrm{~S}$ & Ecotype from NCSU collection & $1800 \mathrm{~S}$ & NCSU, Raleigh, NC & $\mathrm{NA}^{\mathrm{y}}$ \\
\hline 3 & GF2 & 'Raleigh' Mutant & GF2 & NCSU, Raleigh, NC & $\mathrm{NA}^{\mathrm{y}}$ \\
\hline 4 & Seville & Cultivar & S-6-68-516 & $\begin{array}{l}\text { Pursely, Inc.; T.P. Riordan; } \\
\text { O.M. Scotts \& Sons }\end{array}$ & $1978^{x}$ \\
\hline 5 & $\mathrm{C} 1$ & Ecotype from & $\mathrm{C} 1$ & NCSU, Raleigh, NC & $\mathrm{NA}^{\mathrm{y}}$ \\
\hline 6 & $106 \mathrm{~T} 3$ & 'Raleigh' somaclonal variant & $106 \mathrm{~T} 3$ & NCSU, Raleigh, NC & $\mathrm{NA}^{\mathrm{y}}$ \\
\hline
\end{tabular}

$\mathrm{NCSU}=$ North Carolina State University.

${ }^{\text {zBateman, } 1980 .}$

${ }^{\mathrm{y}} \mathrm{NA}=$ Not applicable. Experimental germplasm under development.

${ }^{x}$ Riordan et al., 1980.

Table 2. Percentage of selfed progeny per cross and simple sequence repeat (SSR) markers used to verify hybridity.

\begin{tabular}{lcl}
\hline Cross combinations $^{z}$ & Selfed progeny $(\%)$ & \multicolumn{1}{c}{ SSR markers } \\
\hline $106 \mathrm{~T} 3 \times 1800 \mathrm{~S}$ & 63 & SAM 1709, SAM 23965, SAM 5295 \\
$106 \mathrm{~T} 3 \times \mathrm{C} 1$ & 63 & SAM 5295 \\
$106 \mathrm{~T} 3 \times$ GF 2 & 50 & SAM 21225 \\
$106 \mathrm{~T} 3 \times$ Seville & 63 & SAM 5295, SAM 7483, SAM 12183 \\
$\mathrm{C} 1 \times 1800 \mathrm{~S}$ & 50 & SAM 17792, SAM 21225 \\
$\mathrm{C} 1 \times$ GF 2 & 50 & SAM 21225 \\
$\mathrm{C} 1 \times$ Seville & 63 & SAM 5258, 12183, SAM21225 \\
$\mathrm{GF} 2 \times 1800 \mathrm{~S}$ & 50 & SAM 17792, SAM 7637, SAM 21225 \\
Raleigh $\times 106 \mathrm{~T} 3$ & 63 & SAM 5295 \\
Raleigh $\times 1800 \mathrm{~S}$ & 50 & SAM 17792, SAM 7637, SAM 21225 \\
Raleigh $\times$ C1 & 50 & SAM 21225 \\
Raleigh $\times$ GF2 & 50 & SAM 5295, SAM 21225 \\
Raleigh $\times$ Seville & 63 & SAM 2460, SAM 1512, SAM 11067 \\
Seville $\times 1800 \mathrm{~S}$ & 50 & SAM 434, SAM 1709, SAM 21225 \\
Seville $\times$ GF2 & 63 & SAM 21225, SAM 7637, SAM 4343 \\
\hline A &
\end{tabular}

${ }^{\mathrm{z}} \mathrm{A}$ total of eight crosses were produced per parental combination. separation procedures according to Fisher's protected least significant difference (LSD) values were used to compare parental vs. family means as well as parental vs. self means.

\section{Results and Discussion}

The interaction of location by year was significant for winter survival, spring greenup, and genetic color (Table 3). The significant interaction resulted largely from differences in magnitude between years and locations. For example, winter survival was high at Lake Wheeler in 2012 and extremely low at Laurel Springs in 2013 and 2014. However, moderate shifts in cold response as well as genetic color rankings were identified, and both location and year accounted for a large amount of variation in their models. Environmental variation contributed less than genetic effects to the overall variation in the majority of turf quality traits as expected for qualitative characteristics. Similar results were reported in zoysiagrass (Zoysia spp.) where large environmental influences were identified for stress-related traits but not for turf quality traits (Schwartz et al., 2009). In addition, large environmental and error effects were also identified for genetic color in Schwartz et al. (2009) who suggested that the use of a handheld normalized difference vegetation index sensor, which has been shown to improve the calculation of color heritability (Kenworthy et al., 2006), could increase realized heritability of genetic color by reducing error variation.

Cold response. Significant variability for cold response was identified among the parental lines in this study (Table 3). According to means separation using Fisher's protected LSD, 'Raleigh's' winter survival was significantly better than all other parental lines (Table 4). The remaining five parental lines could not be significantly distinguished from one another in terms of their winter survival. 'Raleigh' was also the only parent with a significant parental effect for winter survival (Table 5). These findings are consistent with previous studies documenting 'Raleigh's' high level of cold tolerance ( $\mathrm{Li}$ et al., 2010; Maier et al., 1994; Moseley et al., 2010; Philley et al., 1988). Genotypes ' $\mathrm{C} 1$ ' and '106T3' had the lowest levels of winter survival across locations and years. Although ' $\mathrm{C} 1$ ' is a collection and thus little is known about its genetic makeup, previous studies have reported varying levels of winter survival for '106T3' (Moseley et al., 2010; Reynolds et al., 2009) indicating that this genotype may not be stable across different environments. Somaclonal variants such as '106T3' can be unpredictable in nature and genetically unstable (Dennis et al., 1987; Kaeppler and Phillips, 1993; Lee and Phillips, 1988), and results suggested that further evaluation of the line is needed. Likewise, 'Seville' had higher winter survival than expected for a known cold susceptible cultivar. Previous studies (Kimball et al., 2016a, 2016b; Moseley 
Table 3. Analysis of variance for the main effects (location, year, parents, selfing, and crosses) and the interactions of a nonreciprocal mating design for winter survival, spring green-up, turf quality, genetic color, leaf texture, turf density, midseason cover, and end-of-season cover from data collected at the Lake Wheeler Turfgrass Field Laboratory in Raleigh, NC, and the Upper Mountain Research Station in Laurel Springs, NC, from 2011 to 2014. Estimations of general combining ability (GCA) and specific combining ability (SCA) were determined among crosses for each parental genotype.

\begin{tabular}{|c|c|c|c|c|c|c|c|c|c|}
\hline Source & df & $\begin{array}{l}\text { Winter } \\
\text { survival }\end{array}$ & $\begin{array}{l}\text { Spring } \\
\text { green-up }\end{array}$ & $\begin{array}{c}\text { Turf } \\
\text { quality }\end{array}$ & $\begin{array}{l}\text { Genetic } \\
\text { color }\end{array}$ & $\begin{array}{c}\text { Leaf } \\
\text { texture }\end{array}$ & $\begin{array}{c}\text { Turf } \\
\text { density }\end{array}$ & $\begin{array}{c}\text { Midseason } \\
\text { cover }\end{array}$ & $\begin{array}{c}\text { End-of-season } \\
\text { cover }\end{array}$ \\
\hline Location & 1 & $577.19 * * *$ & $714.50 * * *$ & $16.43^{* *}$ & 13.39 & $6.79 * *$ & $66.53 * * *$ & $11,058.01 * * *$ & $32,792.54 * * *$ \\
\hline Year & 2 & $746.73 * * *$ & $325.45^{* * *}$ & $56.92 * * *$ & $21.85^{* *}$ & $6.43 * *$ & $39.73 * * *$ & 60.33 & 57.54 \\
\hline Replication & 2 & $13.08 * * *$ & 7.27 & 3.43 & 1.06 & 1.01 & $23.50 * * *$ & $1,980.84 *$ & $8,612.05 * * *$ \\
\hline Parents & 6 & $45.62 * * *$ & $69.49 * * *$ & $32.67 * * *$ & $23.72 * * *$ & $2.45^{*}$ & $54.45 * * *$ & $5,534.68 * * *$ & $6,904.26 * * *$ \\
\hline Selfs & 5 & 3.40 & 5.30 & $20.75 * * *$ & $13.69 * * *$ & $10.68 * * *$ & $31.62 * * *$ & $6,613.43 * * *$ & $5,339.67$ \\
\hline Crosses & 14 & $23.49 * * *$ & $17.29 * * *$ & $14.87 * * *$ & $9.24 * * *$ & $5.31 * * *$ & $20.36 * * *$ & $2,393.88 * * *$ & $4,892.72 * * *$ \\
\hline GCA & 5 & $19.36 * * *$ & $43.09 * * *$ & $27.76 * * *$ & 1.65 & $22.56 * * *$ & $29.81 * * *$ & $4,243.47 * * *$ & $5,214.35 * * *$ \\
\hline SCA & 9 & $30.47 * * *$ & $39.75 * * *$ & $23.48 * * *$ & $6.57 * * *$ & $18.24 * * *$ & $31.83 * * *$ & $1,905.71 * * *$ & $5,425.18 * * *$ \\
\hline Parents vs. crosses & 1 & 0.09 & 0.07 & 4.21 & 0.3 & 0.01 & 5.21 & $1,374.97$ & $2,632.53$ \\
\hline Parents vs. selfs & 1 & $107.64 * * *$ & $257.86 * * *$ & $79.84 * * *$ & 0.08 & $139.77 * * *$ & $116.48 * * *$ & $7,967.81 * * *$ & $12,549.01 * *$ \\
\hline Location $\times$ year & 2 & $141.34 * * *$ & $157.94 * * *$ & 0.25 & $29.32 * * *$ & 0.91 & 4.08 & 200.23 & 221.68 \\
\hline Location $\times$ replication & 2 & 1.66 & 0.41 & 0.64 & $13.82 * *$ & 0.38 & $9.83 *$ & 62.80 & $2,688.82$ \\
\hline Location $\times$ parents & 6 & $22.21 * * *$ & $37.18 * * *$ & $11.82 * * *$ & $11.75 * * *$ & 0.77 & $19.82 * * *$ & $5,413.33 * * *$ & $5,711.20 * * *$ \\
\hline Location $\times$ crosses & 14 & $6.03 * * *$ & 5.19 & $5.58 * *$ & 2.41 & $3.06 * * *$ & $7.31 * *$ & 681.03 & $3,745.14 * * *$ \\
\hline Year $\times$ replication & 4 & 1.48 & 3.86 & 0.85 & 1.71 & 1.06 & 0.34 & 203.46 & 212.16 \\
\hline Year $\times$ parents & 12 & $17.63 * * *$ & $18.58 * * *$ & 2.70 & 2.25 & $6.97 * * *$ & 1.76 & $2,210.54 *$ & $2,323.79 *$ \\
\hline Year $\times$ crosses & 28 & $6.51 * * *$ & 5.13 & 2.75 & 2.35 & 0.82 & 3.2 & $1,149.62$ & $1,325.51$ \\
\hline Location $\times$ year $\times$ replication & 4 & $4.69 *$ & 2.48 & 0.44 & 1.6 & 1.48 & $15.81 * *$ & $2,348.05$ & $2,437.01$ \\
\hline Location $\times$ year $\times$ parents & 12 & $8.30 * * *$ & $20.46 * * *$ & $6.47 * *$ & 1.7 & 1.73 & 2.16 & $1,578.49$ & $1,696.39$ \\
\hline Location $\times$ year $\times$ crosses & 28 & $4.67 * * *$ & 2.53 & 2.11 & 2.63 & 0.58 & 1.52 & 795.35 & 847.25 \\
\hline
\end{tabular}

$* P<0.05, * * P<0.01, * * * P<0.0001$.

Table 4. Performance of six st. augustinegrass parents and their cross combinations for winter survival, spring green-up, turf quality, genetic color, leaf texture, and turf density (rated on a 1-9 scale), and midseason cover and end-of-season cover (rated on a $0 \%$ to $100 \%$ scale) from data collected at the Lake Wheeler Turfgrass Field Laboratory in Raleigh, NC, and the Upper Mountain Research Station in Laurel Springs, NC, from 2011 to 2014.

\begin{tabular}{|c|c|c|c|c|c|c|c|c|c|}
\hline ID & $\begin{array}{c}\text { No. of } \\
\text { lines }\end{array}$ & $\begin{array}{c}\text { Winter } \\
\text { survival }\end{array}$ & $\begin{array}{c}\text { Spring } \\
\text { green-up }\end{array}$ & $\begin{array}{c}\text { Turf } \\
\text { quality }\end{array}$ & $\begin{array}{c}\text { Genetic } \\
\text { color }\end{array}$ & $\begin{array}{c}\text { Leaf } \\
\text { texture }\end{array}$ & $\begin{array}{c}\text { Turf } \\
\text { density }\end{array}$ & $\begin{array}{c}\text { Midseason } \\
\text { cover }\end{array}$ & $\begin{array}{c}\text { End-of-season } \\
\text { cover }\end{array}$ \\
\hline Raleigh & 4 & $4.86 \mathrm{a}^{\mathrm{z}}$ & $2.96 \mathrm{ab}$ & $6.39 \mathrm{bcd}$ & $6.61 \mathrm{bc}$ & $5.9 \mathrm{efg}$ & $6.27 \mathrm{bcd}$ & $78.96 \mathrm{cdef}$ & $93.96 \mathrm{ab}$ \\
\hline $1800 \mathrm{~S}$ & 4 & $3.56 \mathrm{cde}$ & $2.79 \mathrm{ab}$ & $6.64 \mathrm{~b}$ & $6.87 \mathrm{~b}$ & $6.58 \mathrm{ab}$ & $6.62 \mathrm{bc}$ & $64.58 \mathrm{ghi}$ & 85.42 abcd \\
\hline GF2 & 4 & $3.46 \mathrm{cde}$ & $3.75 \mathrm{a}$ & 6.26 bcde & $6.45 \mathrm{bcd}$ & $6.11 \mathrm{cdef}$ & $5.87 \mathrm{cdef}$ & $54.79 \mathrm{ij}$ & 80.94 bcde \\
\hline Seville & 4 & $3.46 \mathrm{cde}$ & $1.64 \mathrm{cde}$ & $7.56 \mathrm{a}$ & $7.43 \mathrm{a}$ & 6.27 abcdef & $7.58 \mathrm{a}$ & $98.96 \mathrm{a}$ & $87.29 \mathrm{abcd}$ \\
\hline $\mathrm{C} 1$ & 4 & $3.24 \mathrm{defg}$ & $2.31 \mathrm{bcd}$ & 5.48 fghi & $5.97 \mathrm{defg}$ & $5.89 \mathrm{efg}$ & 5.05 fghi & $57.93 \mathrm{hij}$ & $71.35 \mathrm{efgh}$ \\
\hline $106 \mathrm{~T} 3$ & 4 & $3.08 \mathrm{efgh}$ & $2.18 \mathrm{bcd}$ & $5.06 \mathrm{ghi}$ & $6.01 \mathrm{def}$ & $6.51 \mathrm{abc}$ & $4.62 \mathrm{ghi}$ & $44.79 \mathrm{j}$ & $64.17 \mathrm{gh}$ \\
\hline $106 \mathrm{~T} 3 \times 1800 \mathrm{~S}$ & 3 & $3.61 \mathrm{~cd}$ & $2.29 \mathrm{bcd}$ & $5.65 \mathrm{efg}$ & $5.96 \mathrm{defg}$ & $5.87 \mathrm{fg}$ & $5.28 \mathrm{efg}$ & $89.44 \mathrm{abc}$ & 80 cde \\
\hline $106 \mathrm{~T} 3 \times \mathrm{C} 1$ & 3 & $2.66 \mathrm{hij}$ & $1.5 \mathrm{cde}$ & $4.88 \mathrm{hi}$ & $5.64 \mathrm{fg}$ & $5.33 \mathrm{~h}$ & $4.48 \mathrm{hi}$ & 73.33 defgh & $63.47 \mathrm{gh}$ \\
\hline $106 \mathrm{~T} 3 \times \mathrm{GF} 2$ & 4 & $3.57 \mathrm{cde}$ & $2.32 \mathrm{bc}$ & $5.93 \mathrm{def}$ & $6.4 \mathrm{bcd}$ & $6.55 \mathrm{ab}$ & $5.59 \mathrm{def}$ & 79.49 cdef & $76.88 \mathrm{def}$ \\
\hline $106 \mathrm{~T} 3 \times$ Seville & 3 & $3.8 \mathrm{bc}$ & $2.29 \mathrm{bcd}$ & $6.54 \mathrm{bc}$ & $6.65 \mathrm{bc}$ & 6.17 bcdef & $6.83 \mathrm{~b}$ & $90.28 \mathrm{abc}$ & $95.69 \mathrm{a}$ \\
\hline $\mathrm{C} 1 \times 1800 \mathrm{~S}$ & 4 & $4.22 \mathrm{~b}$ & $2.68 \mathrm{~b}$ & $6.68 \mathrm{~b}$ & $6.73 \mathrm{bc}$ & $6.57 \mathrm{ab}$ & $6.55 \mathrm{bc}$ & 79.79 cde & $90 \mathrm{abc}$ \\
\hline $\mathrm{C} 1 \times \mathrm{GF} 2$ & 4 & $2.28 \mathrm{j}$ & $1.35 \mathrm{ed}$ & - & $4.85 \mathrm{~h}$ & $5.67 \mathrm{gh}$ & $4.29 \mathrm{i}$ & 76.46 defg & $61.67 \mathrm{~h}$ \\
\hline C1 $\times$ Seville & 3 & $2.65 \mathrm{hij}$ & $1.04 \mathrm{e}$ & $5.69 \mathrm{efg}$ & $5.39 \mathrm{~g}$ & $6.06 \mathrm{defg}$ & $5.21 \mathrm{efgh}$ & $95.83 \mathrm{ab}$ & 75.83 defg \\
\hline $\mathrm{GF} 2 \times 1800 \mathrm{~S}$ & 4 & $2.81 \mathrm{ghi}$ & $1.76 \mathrm{cde}$ & $5.35 \mathrm{gh}$ & $6.08 \mathrm{def}$ & $5.98 \mathrm{efg}$ & $5.12 \mathrm{fgh}$ & 66.75 fghi & $66.17 \mathrm{fgh}$ \\
\hline Raleigh $\times 106 \mathrm{~T} 3$ & 3 & 3.31 cdef & $1.93 \mathrm{cde}$ & $5.97 \mathrm{cdef}$ & $5.88 \mathrm{efg}$ & $6.4 \mathrm{abcd}$ & $5.75 \mathrm{def}$ & $83.06 \mathrm{bcd}$ & $86.81 \mathrm{abcd}$ \\
\hline Raleigh $\times 1800 \mathrm{~S}$ & 4 & 2.92 fghi & $1.63 \mathrm{cde}$ & $5.13 \mathrm{gh}$ & $5.76 \mathrm{fg}$ & $5.44 \mathrm{~h}$ & $4.73 \mathrm{ghi}$ & $69.58 \mathrm{efgh}$ & 75.42 defg \\
\hline Raleigh $\times \mathrm{C} 1$ & 4 & $3.08 \mathrm{efgh}$ & $2.13 \mathrm{bcd}$ & $5.02 \mathrm{hi}$ & $5.93 \mathrm{defg}$ & $6.1 \mathrm{def}$ & $4.85 \mathrm{ghi}$ & 72.71 defgh & $68.75 \mathrm{efgh}$ \\
\hline Raleigh $\times$ GF2 & 4 & $2.74 \mathrm{hi}$ & $1.54 \mathrm{cde}$ & $5.32 \mathrm{gh}$ & $5.79 \mathrm{efg}$ & $5.99 \mathrm{efg}$ & $5.24 \mathrm{efgh}$ & $56.41 \mathrm{ij}$ & $66.46 \mathrm{fgh}$ \\
\hline Raleigh $\times$ Seville & 3 & 2.87 gfghi & $1.43 \mathrm{cde}$ & $6.49 \mathrm{bcd}$ & $6.29 \mathrm{cde}$ & $6.6 \mathrm{a}$ & 5.89 cde & $90.83 \mathrm{abc}$ & $77.5 \mathrm{def}$ \\
\hline Seville $\times 1800 \mathrm{~S}$ & 4 & $2.49 \mathrm{ij}$ & $1.49 \mathrm{cde}$ & 5.64 fghi & $6.02 \mathrm{def}$ & $6.31 \mathrm{abcde}$ & $5.18 \mathrm{efgh}$ & 75.42 defg & 69.46 efgh \\
\hline Seville $\times$ GF2 & 3 & $2.46 \mathrm{ij}$ & $1.02 \mathrm{e}$ & $6.59 \mathrm{~b}$ & $6.82 \mathrm{bc}$ & $6.66 \mathrm{a}$ & $6.03 \mathrm{~cd}$ & 80.28 cde & $68.61 \mathrm{efgh}$ \\
\hline
\end{tabular}

${ }^{\mathrm{z}}$ Means with the same letter are not significantly different from one another.

et al., 2010; Philley et al., 1995) have reported varying winterkill and freeze damage in 'Seville' and suggest that 'Seville' is another genotype whose performance is not consistent across environments.

For spring green-up, 'GF2', 'Raleigh', and ' 1800 S' were the top performers. 'Seville' was the slowest to green-up. As the best and worst performers, 'GF2' and 'Seville' had significant parental effects for spring green-up, respectively (Table 5). A significant, positive correlation (0.63) between winter survival and spring green-up was detected between parental lines as well as between crosses in this study (Table 6) indicating that a genotype able to withstand cold temperatures in winter will green up faster in the spring than a genotype severely affected by winterkill. Little has been reported on st. augustinegrass' rate of spring green-up in the literature with the exception of limited data from the NTEP (2012).

Across all locations and years, the GCA and SCA effects were both significant sources of variation for winter survival and spring green-up (Table 3). Significant GCA effects indicated parents of genotypes contributed differently to responses in hybrid combinations as well as the involvement of additive gene effects in response to winter survival and spring green-up. However, for winter survival, SCA effects accounted for three times $(\approx 60 \%)$ more of the total genotypic variance than GCA effects $(\approx 20 \%)$. The high
SCA effects for winter survival suggested that nonadditive gene effects (i.e., dominance and epistasis) play an important role in cold response in the species. For example, while 'Raleigh' had the largest parental effect for winter survival $(0.82)$, it had a significant negative GCA effect $(-0.72)$ and a nonsignificant, mainly neutral self-effect $(-0.05)$ (Table 5). Because 'Raleigh' was no longer distinguishable from other parents on selfing, these results suggest that while dominance could contribute to winter survival, strong heterotic effects and epistatic interactions could also play a key role in the genetic control of this trait. Conversely, Philley et al. (1998) found that the largest portion of variance was additive for lethal temperature 
Table 5. Estimates of parental, self, and general combining ability (GCA) effects for winter survival, spring green-up, turf quality, genetic color, leaf texture, turf density, midseason cover, and end-of-season cover from data collected at the Lake Wheeler Turfgrass Field Laboratory in Raleigh, NC, and the Upper Mountain Research Station in Laurel Springs, NC, from 2011 to 2014.

\begin{tabular}{|c|c|c|c|c|c|c|c|c|c|c|c|c|}
\hline \multirow{2}{*}{ a. } & \multicolumn{3}{|c|}{ Winter survival } & \multicolumn{3}{|c|}{ Spring green-up } & \multicolumn{3}{|c|}{ Midseason cover } & \multicolumn{3}{|c|}{ End-of-season cover } \\
\hline & $\mathrm{P}$ & $\mathrm{S}$ & GCA & $\mathrm{P}$ & $\mathrm{S}$ & GCA & $\mathrm{P}$ & $\mathrm{S}$ & GCA & $\mathrm{P}$ & $\mathrm{S}$ & GCA \\
\hline Raleigh & $0.82 * * *$ & -0.05 & $-0.72 * * *$ & 0.08 & -0.02 & $-1.26^{* * *}$ & 0.66 & $7.94 * *$ & $8.29^{*}$ & 5.76 & -2.39 & -6.72 \\
\hline $106 \mathrm{~T} 3$ & -0.19 & -0.09 & 0.05 & -0.34 & -0.05 & -0.23 & $-19.62 * * *$ & $-9.73 * *$ & $10.01 * * *$ & $-10.35^{*}$ & $-8.57^{*}$ & 2.10 \\
\hline $\mathrm{C} 1$ & -0.33 & $0.24 * *$ & $-0.51 * * *$ & -0.30 & 0.15 & -0.70 & -1.06 & 3.57 & $9.20 * *$ & -5.35 & $6.33 *$ & $-8.96^{*}$ \\
\hline Seville & -0.14 & 0.01 & $0.96 * * *$ & $-1.25 * * *$ & -0.20 & $1.45^{* * *}$ & $26.77 * * *$ & $17.48 * * *$ & -2.96 & 5.35 & $10.33 * * *$ & $21.79 * * *$ \\
\hline GF2 & -0.09 & -0.06 & $0.66 * *$ & $1.56 * * *$ & -0.22 & $1.71 * * *$ & -8.23 & 3.22 & $-35.27 * * *$ & 0.63 & 1.63 & -3.40 \\
\hline $1800 \mathrm{~S}$ & -0.06 & -0.06 & $-0.44 * *$ & 0.25 & 0.32 & $-0.97 * * *$ & 1.49 & $-22.48 * * *$ & $10.73 * *$ & 3.96 & -7.34 & -4.81 \\
\hline b. & \multicolumn{3}{|c|}{ Turf quality } & \multicolumn{3}{|c|}{ Genetic color } & \multicolumn{3}{|c|}{ Leaf texture } & \multicolumn{3}{|c|}{ Turf density } \\
\hline Line & $\mathrm{P}$ & $\mathrm{S}$ & GCA & $\mathrm{P}$ & $\mathrm{S}$ & GCA & $\mathrm{P}$ & $\mathrm{S}$ & GCA & $\mathrm{P}$ & $\mathrm{S}$ & GCA \\
\hline Raleigh & -0.23 & $-0.36^{*}$ & $-0.95 * * *$ & -0.06 & $-0.36^{*}$ & $-0.97 * * *$ & $-0.38^{*}$ & $-0.33 * *$ & -0.11 & -0.20 & $-0.38^{*}$ & $-0.98 * * *$ \\
\hline $106 \mathrm{~T} 3$ & -0.69 & -0.23 & $-0.24 *$ & $-0.48^{*}$ & 0.05 & $-0.31 * * *$ & $0.35^{*}$ & 0.12 & -0.06 & $-0.96 * *$ & $-0.51 *$ & -0.17 \\
\hline $\mathrm{C} 1$ & -0.53 & -0.01 & $-0.74 * * *$ & $-0.54^{*}$ & $-0.17^{*}$ & $-0.77 * * *$ & $-0.35^{*}$ & $-0.16^{*}$ & -0.16 & $-0.64 *$ & -0.11 & $-0.78 * * *$ \\
\hline Seville & $1.20 * * *$ & $0.91 * * *$ & $2.07 * * *$ & $1.02 * * *$ & $0.56 * * *$ & $1.67 * * *$ & 0.09 & $0.68 * * *$ & $0.48 * *$ & $1.44 * * *$ & $1.04 * * *$ & $2.10 * * *$ \\
\hline GF2 & -0.09 & 0.02 & $0.63 *$ & -0.18 & -0.12 & $1.14 * * *$ & -0.03 & 0.06 & 0.06 & -0.14 & -0.01 & $0.67 *$ \\
\hline $1800 \mathrm{~S}$ & 0.35 & -0.33 & $-0.77 * * *$ & 0.24 & 0.04 & $-0.76 * * *$ & $0.33 *$ & $-0.36^{*}$ & -0.22 & 0.50 & -0.03 & $-0.83 * *$ \\
\hline
\end{tabular}

$\mathrm{P}=$ parental effect; $\mathrm{S}=$ self-effect.

$* P<0.05, * * P<0.01, * * * P<0.0001$.

Table 6. Pearson correlation coefficients between parental lines and between crosses for winter survival, spring green-up, turf quality, leaf texture, genetic color, turf density, midseason cover, and end-ofseason cover from data collected at the Lake Wheeler Turfgrass Field Laboratory in Raleigh, NC, and the Upper Mountain Research Station in Laurel Springs, NC, from 2011 to 2014.

\begin{tabular}{|c|c|c|c|c|c|c|c|}
\hline & $\begin{array}{l}\text { Winter } \\
\text { survival }\end{array}$ & $\begin{array}{c}\text { Spring } \\
\text { green-up }\end{array}$ & $\begin{array}{c}\text { Turf } \\
\text { quality }\end{array}$ & $\begin{array}{c}\text { Leaf } \\
\text { texture }\end{array}$ & $\begin{array}{l}\text { Genetic } \\
\text { color }\end{array}$ & $\begin{array}{c}\text { Turf } \\
\text { density }\end{array}$ & $\begin{array}{c}\text { Midseason } \\
\text { cover }\end{array}$ \\
\hline \multicolumn{8}{|l|}{ Parental lines } \\
\hline Spring green-up & $0.49 * *$ & & & & & & \\
\hline Turf quality & $-0.49 * *$ & 0.26 & & & & & \\
\hline Leaf texture & -0.28 & -0.17 & $0.54 * *$ & & & & \\
\hline Genetic color & -0.02 & 0.00 & $0.50 * *$ & 0.14 & & & \\
\hline Turf density & -0.26 & 0.21 & $0.83 * * *$ & $0.56 * *$ & 0.29 & & \\
\hline Midseason cover & -0.46 & $0.55^{*}$ & $0.80 * * *$ & 0.25 & $0.67 * *$ & $0.73 * *$ & \\
\hline End-of-season cover & -0.30 & 0.07 & $0.52 * *$ & 0.09 & $0.54 * * *$ & $0.47 * *$ & $0.83 * * *$ \\
\hline \multicolumn{8}{|l|}{ Crosses } \\
\hline Spring green-up & $0.79 * * *$ & & & & & & \\
\hline Turf quality & $0.11 *$ & $0.22 * * *$ & & & & & \\
\hline Leaf texture & $-0.10 *$ & -0.06 & $0.30 * * *$ & & & & \\
\hline Genetic color & 0.06 & 0.06 & $0.48 * * *$ & 0.13 & & & \\
\hline Turf density & $0.44 * * *$ & $0.44 * * *$ & $0.79 * * *$ & $0.19 * * *$ & $0.38 * * *$ & & \\
\hline Midseason cover & $0.55 * * *$ & $0.46^{* * *}$ & $0.44 * * *$ & 0.08 & -0.03 & $0.58 * * *$ & \\
\hline End-of-season cover & $0.72 * * *$ & $0.56^{* * *}$ & $0.37 * * *$ & -0.02 & $0.22 * * *$ & $0.67 * * *$ & $0.55 * * *$ \\
\hline
\end{tabular}

and winter survival, suggesting that st. augustinegrass should respond well to selection. The differences in results of the two studies may reflect the large environmental differences between testing regions as well as the germplasm used. In addition, this study has demonstrated (see below under selfing rates section) the importance of confirming true hybridity in crosses of st. augustinegrass as selfing is an important issue to consider in these types of studies.

There was a large range of significant positive and negative SCA effects for winter survival and spring green-up for the majority of crosses (Table 7). In general, SCA estimates indicated that crosses with good winter survival also had faster spring greenup, which is consistent with the high correlations identified between family means for these traits (Table 6). The cross 'Seville' $\times$ 'GF2' had larger SCA effects for winter survival and spring green-up than any other cross (Table 7). The effect of this particular cross largely contributed to the significantly high SCA effects identified for winter survival and spring green-up and provide evidence that a more complex type of inheritance than additive gene effects may be involved.

Turf quality. Significant variability for turf quality traits was identified among the parental lines in this study (Table 3). Although ' $1800 \mathrm{~S}$ ' had the finest leaf texture, 'Seville' outperformed all parental lines in turf quality, turf density, and genetic color (Table 4). Turf quality provided excellent separation of the genotypes (Table 3 ) with the most winter hardy genotypes forming two separate groups based on Fisher's LSD values. The genotypes 'Raleigh', '1800S', and 'GF2' appeared to have an intermediate turf quality between 'Seville' and the lowest parental performers, 'C1' and '106T3'. Similar results can be seen for genetic color and turf density, while, as expected, leaf texture had the lowest amount of variability between the parental lines (Table 4).

Across all locations and years, GCA and SCA effects were significant sources of variation for turf quality traits (Table 3). Additive gene effects appear to play a larger role than nonadditive effects for turf quality and leaf texture, while SCA or nonadditive effects contributed twice as much to the overall variation than GCA effects for genetic color (Table 3). The magnitude of SCA effects was slightly higher than GCA effects for turf density as well. These results indicated that while turf quality traits can be improved through selecting parents with significant GCA values, progeny testing of specific parental combinations could provide more value to our breeding program. For example, the cross 'Seville' $\times$ 'GF2' had the largest SCA values for turf quality, genetic color, and turf density along with high SCA values for winter survival and spring green-up (Table 7), and has proven to be an excellent st. augustinegrass cross producing progenies with superior performance.

The cultivar Seville had the highest positive GCA effect for all turf quality traits (Table 5). The majority of winter hardy genotypes provided a negative or neutral GCA effect, with the exception of ' $\mathrm{GF} 2$ ' on overall turf quality, genetic color, and turf density. 'GF2' also had a positive GCA effect for both winter survival and spring green-up. Previous studies have reported better establishment rates, qualitative characteristics, and similar winter survival of 'GF2' when compared with 'Raleigh' (Li, 2007; Reynolds et al., 2009). This genotype showed the most promise as a parental line to transfer both winterhardiness and turf quality traits into new breeding lines, especially with the low correlations identified between these two types of traits (Table 6).

Establishment. Significant variability for midseason and end-of-season cover ratings was identified among the parental lines in this study (Table 3). For midseason cover, 'Seville' had significantly better cover than other parental lines at $99 \%$, which dropped to $\approx 87 \%$ at the end of the season (Table 4 ). This may indicate that low temperature injury was already beginning to occur in 'Seville' during the fall, especially as it was the only genotype whose cover ratings dropped between rating times. 'Raleigh' had the second 
Table 7. Estimates of the specific combining ability of 15 crosses in st. augustinegrass for winter survival, spring green-up, turf quality, leaf texture, genetic color, turf density, midseason cover, and end-of-season cover traits evaluated at the Lake Wheeler Turfgrass Field Laboratory in Raleigh, NC, and the Upper Mountain Research Station in Laurel Springs, NC, from 2011 to 2014.

\begin{tabular}{|c|c|c|c|c|c|c|c|c|}
\hline Cross & $\begin{array}{l}\text { Winter } \\
\text { survival }\end{array}$ & $\begin{array}{l}\text { Spring } \\
\text { green-up }\end{array}$ & $\begin{array}{c}\text { Turf } \\
\text { quality }\end{array}$ & $\begin{array}{l}\text { Leaf } \\
\text { texture }\end{array}$ & $\begin{array}{l}\text { Genetic } \\
\text { color }\end{array}$ & $\begin{array}{c}\text { Turf } \\
\text { density }\end{array}$ & $\begin{array}{c}\text { Midseason } \\
\text { cover }\end{array}$ & $\begin{array}{c}\text { End-of-season } \\
\text { cover }\end{array}$ \\
\hline $106 \mathrm{~T} 3 \times 1800 \mathrm{~s}$ & $0.43^{*}$ & $0.75 * *$ & 0.27 & -0.04 & 0.29 & 0.18 & -0.19 & 2.00 \\
\hline $106 \mathrm{~T} 3 \times \mathrm{C} 1$ & $-0.99 * * *$ & $-1.03 * *$ & $-1.23 * * *$ & $-0.75 * * *$ & $-0.90 * * *$ & $-1.51 * * *$ & -7.00 & $-19.49 * * *$ \\
\hline $106 \mathrm{~T} 3 \times \mathrm{GF} 2$ & -0.04 & -0.19 & -0.21 & $0.4^{* *}$ & -0.06 & -0.36 & -0.06 & -6.12 \\
\hline $106 \mathrm{~T} 3 \times$ Seville & 0.18 & -0.21 & 0.40 & 0.02 & 0.24 & $0.90 * *$ & $11.38^{*}$ & $12.89 * *$ \\
\hline $\mathrm{C} 1 \times 1800 \mathrm{~S}$ & $0.43^{*}$ & $0.75^{* *}$ & 0.27 & -0.04 & 0.29 & 0.18 & -0.19 & 2.00 \\
\hline $\mathrm{C} 1 \times \mathrm{GF} 2$ & $-1.00 * * *$ & $-0.91 * *$ & $-0.99 * * *$ & -0.25 & $-1.00 * * *$ & $-1.01 * *$ & 6.63 & $-12.99 * *$ \\
\hline $\mathrm{C} 1 \times$ Seville & $-0.41 *$ & $-1.00 * *$ & 0.05 & 0.01 & $-0.57 * *$ & -0.08 & $17.74 * *$ & 4.09 \\
\hline $\mathrm{GF} 2 \times 1800 \mathrm{~S}$ & $-1.65 * * *$ & $-2.63 * * *$ & $-1.33 * *$ & $-1.13 * * *$ & $-1.62 * * *$ & $-1.57 * *$ & $21.67^{*}$ & -14.14 \\
\hline Raleigh $\times 106 \mathrm{~T} 3$ & $0.42 *$ & $0.68 * *$ & $0.78 * * *$ & $0.37 *$ & $0.43 *$ & $0.80 * *$ & -4.14 & $10.72 *$ \\
\hline Raleigh $\times 1800 \mathrm{~S}$ & $0.51 * *$ & $1.11^{* *}$ & $0.51 *$ & $-0.40 *$ & $0.76^{* *}$ & 0.46 & $-17.06 * *$ & 6.65 \\
\hline Raleigh $\times \mathrm{C} 1$ & $0.78 * * *$ & $1.34 * * * *$ & 0.35 & 0.21 & $0.94 * * *$ & $0.54 *$ & $-11.01 *$ & 4.63 \\
\hline Raleigh $\times$ GF2 & $-0.77 * *$ & $-1.63 * * *$ & $-0.59 *$ & -0.17 & $-0.99 * * *$ & -0.47 & $15.6 * *$ & -3.72 \\
\hline Raleigh $\times$ Seville & $-0.94 * * *$ & $-1.5 * * *$ & $-1.05 * * *$ & -0.01 & $-1.15 * * *$ & $-1.33 * * *$ & $16.61 * *$ & $-18.28 * *$ \\
\hline Seville $\times 1800 \mathrm{~S}$ & $-1.60 * * *$ & $-1.74 * * *$ & $-1.9 * * *$ & -0.19 & $-1.57 * * *$ & $-2.01 * * *$ & 0.03 & $-26.98 * * *$ \\
\hline Seville $\times$ GF2 & $2.77 * * *$ & $4.46 * * *$ & $2.50 * * *$ & 0.18 & $3.05 * * *$ & $2.53 * * *$ & $-45.76^{* * *}$ & $28.28 * *$ \\
\hline
\end{tabular}

$* P>0.05, * * P>0.01, * * * P>0.0001$.

highest cover rating during midseason and the best cover rating at the end of the season (Table 4). Interestingly, significant positive correlations were found between winter survival and both midseason (0.55) and end-ofseason (0.72) cover ratings when comparing crosses but nonsignificant, negative correlations between these traits were identified when comparing parental lines (Table 6). It appears that these negative correlations between parental traits were most likely attributed to 'Seville's' high cover ratings and intermediate winter survival (Table 4). The positive correlations between winter survival and cover ratings when comparing crosses indicated that a full turf stand, which is indicative of good overall plant health, appeared to aid a genotype's ability to withstand cold temperatures. In addition, full midseason cover indicates fast establishment rates leading to reduced soil exposure for weed competition in newly established turf stands (Busey and Myers, 1979), which is important for new cultivars in the turfgrass industry.

Across all locations and years, GCA and SCA effects were both significant sources of variation for both cover ratings (Table 3). Large positive and negative differences in GCA effects were identified for parental lines. 'Seville' had the largest GCA effect for end-of-season cover followed by '106T3'. '1800S', '106T3', 'C1', and 'Raleigh' which had significantly large GCA effects on midseason cover but not for end-of-season cover (Table 5).

Overall cross performance. As mentioned above, the cross 'Seville' $\times$ 'GF2' proved to be an excellent cross with high performance (Table 4) and significantly high SCA effects (Table 7) for a majority of traits. The crosses '106T3' × '1800S', '106T3' × 'GF2', and '106T3' $\times$ 'Seville' also had good winter survival, fast spring green-up, good turf quality, and fast establishment rates (Table 4). In addition, the cross ' $\mathrm{C} 1$ ' $\times$ ' $1800 \mathrm{~S}$ ' had very good spring green-up and turf quality. Cross combinations with poor performance were identified as well. The cross 'Raleigh' $\times$ 'GF2' produced low performing progenies across
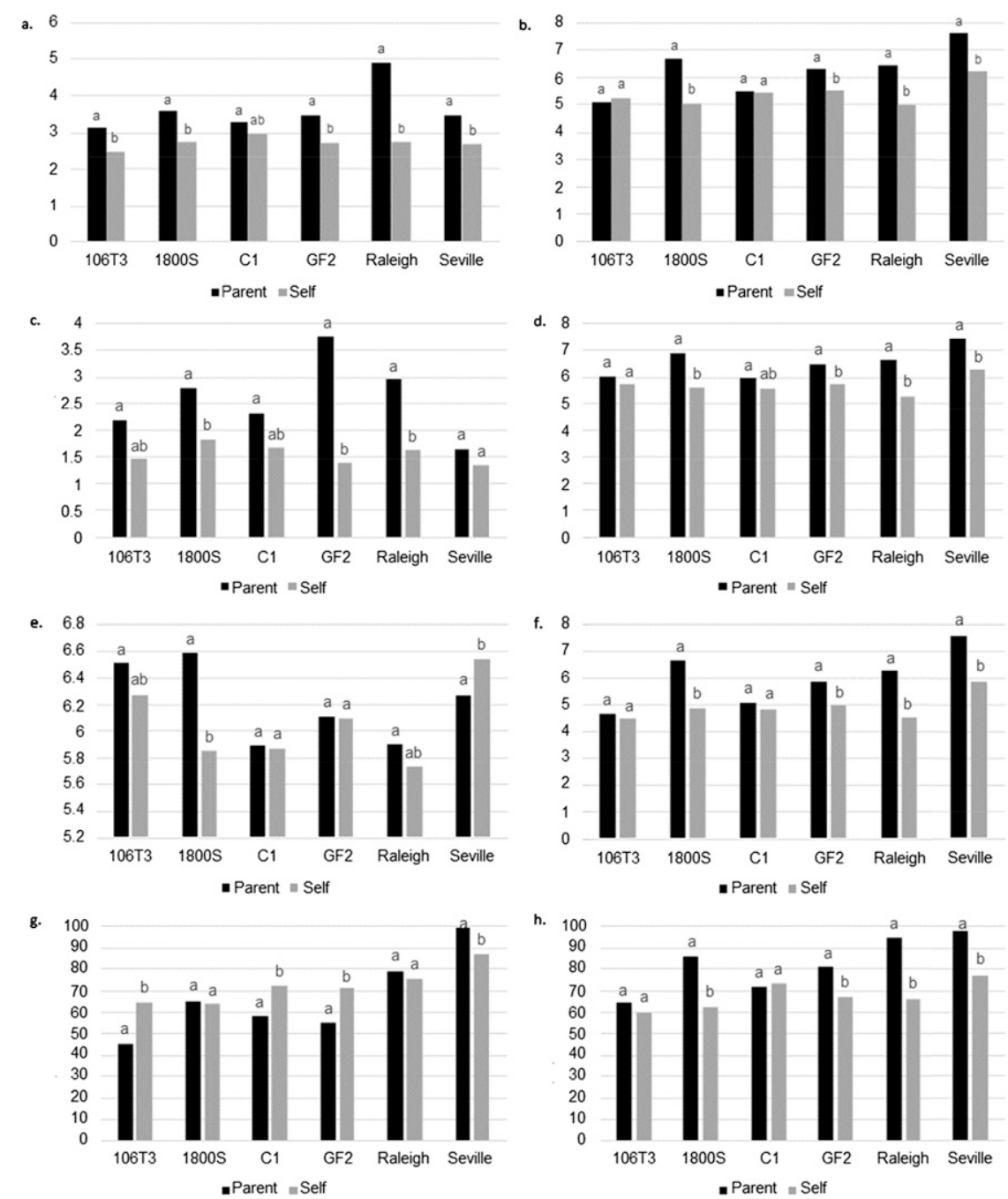

Fig. 1. Least significant means and mean separation between parental and selfed lines for a = winter survival; $b=$ turf quality, spring green-up, genetic color, leaf texture, turf density, midseason cover, and end-of-season cover.

the winter survival and turf quality traits. As 'GF2' is a gamma-ray derived mutant of ' $\mathrm{Ra}$ leigh', it is possible that these two parents are highly similar to one another genetically resulting in high levels of inbreeding depression within crosses between the two.

Selfing rates and inbreeding depression. Although st. augustinegrass is widely known as a predominately outcrossing species, this 
research identified high rates of selfing using SSR markers to confirm parentage. This reflects the importance of using molecular markers to validate the true hybridity of crosses. Studies in common bermudagrass [C. dactylon (L.) Pers. var. dactylon (Tan et al., 2015)] and zoysiagrass (Zoysia spp.) (Guo et al., 2014) have identified high outcrossing rates in these species with the use of molecular markers. However, crosses in these studies were generated in open-pollinated fields where pollen was not limited. The high selfing rates observed in controlled crosses in the current study (Table 2) may be the result of a deficiency in crossing protocols, but could also indicate an underestimation in the levels of self-pollination occurring in the species, especially when pollination is limited to a turf stand of the same genotype. It is important to ascertain the cause of these high selfing rates of controlled crosses as they can negatively affect the progress and efficiency of a breeding program.

Previous studies have reported the negative impacts of inbreeding in st. augustinegrass (Atilano and Busey 1983; Busey, 2003). Comparison of the parental and self means and effects indicate that, in general, inbreeding depression impacted most genotypes negatively across traits (Fig. 1). However, depending on the trait, the selfed progeny of some parents showed little to no change, and a few actually showed improvement. For winter survival, all parental means were significantly higher than their selfed counterparts with the exception of ' $\mathrm{C} 1$ ', indicating that selfing had a negative impact on winter survival of progeny for most parents (Fig. 1A). Similar results were seen for spring green-up, turf quality traits, and establishment (Fig. 1). Little change was seen in the leaf texture of parental lines and their selfs.

Interestingly, ' $\mathrm{C} 1$ ' and '106T3' progeny after selfing improved for all traits except for leaf texture in '106T3' selfs (Table 7). Progeny from neither parental genotype was severely affected by selfing and selfed progeny performed better for several traits (Fig. 1). Although ' $\mathrm{C} 1$ ' is an ecotype and little is known about its historical pedigree, as a somaclonal variant of 'Raleigh', '106T3's' improvement upon selfing provides further evidence that the genotype is genetically unstable. This also indicates that selfing may help stabilize somaclonal variants in st. augustinegrass and improve their performance in the field for important turf quality traits.

\section{Conclusions}

Significant variation was observed between parental lines and the progeny means of crosses for cold response and turf quality traits. The GCA and SCA effects were both significant for all traits, whereas SCA effects were greater for winter survival, genetic color, turf density, and end-of-season cover indicating that nonadditive gene effects may be more important than additive gene effects for these traits. Although 'Raleigh' exhibited a negative GCA effect for winter survival, 'GF2' had a positive GCA effect for both this trait and turf quality and shows promise as a parental line for future breeding efforts in North Carolina.

\section{Literature Cited}

Atilano, R.A. and P. Busey. 1983. Susceptibility of St. Augustinegrass germplasm to Pyricularia grisea. Plant Dis. 67:782-785.

Bateman, D.R. 1980. Notice to sod producers and growers relative to the naming and release of the new St. Augustinegrass cultivar 'Raleigh'. N. C. Ag. Res. Serv., Raleigh, NC.

Busey, P. 2003. Winter survival of St. Augustinegrass cultivars. HortScience 38:1439-1440.

Busey, P. and B.J. Myers. 1979. Growth rates of turfgrasses propagated vegetatively. Agron. J. $71: 817-821$

Dennis, E.S., R.I.S. Brettel, and W.J. Peacock. 1987. A tissue culture induced Adhl null mutant of maize results from a single base change. Mol. Gen. Genet. 210:181-183.

Genovesi, A.D., R.W. Jessup, M.C. Engelke, and B.L. Burson. 2009. Interploid St. Augustinegrass [Stenotaphrum secundatum (Walt.) Kuntze] hybrids recovered by embryo rescue. In Vitro Cell. Dev. Bio. Plant 45:659-666.

Griffing, B. 1956. Concept of general and specific combining ability in relation to diallel crossing systems. Austral. J. Bio. Sci. 9:463-493.

Guo, H.L., D.D. Xue, X. Chen, Y.Q. Zheng, Z.Y. Wang, and J.X. Liu. 2014. Application of SRAP molecular markers for the identification of zoysiagrass hybrids. Acta Hort. 1035:223-232.

Kaeppler, S.M. and R.L. Phillips. 1993. Tissue culture induced DNA methylation variation in maize. Proc. Natl. Acad. Sci. USA 90:8773-8776.

Kenworthy, K.E., C.M. Taliaferro, B.F. Carver, D.L. Martin, J.A. Anderson, and G.E. Bell. 2006 Genetic variation in Cynodon transvaalensis Burtt-Davy. Crop Sci. 46:2376-2381.

Kimball, J.A., T.D. Tuong, C. Arellano, D.P. Livingston, III, and S.R. Milla-Lewis. 2016a. Freeze-testing in St. Augustinegrass I: Evaluation methods. Eur. J. Agron. (In review).

Kimball, J., T.D. Tuong, C. Arellano, D.P. Livingston, III, and S.R. Milla-Lewis. 2016b. Freeze-testing in St. Augustinegrass II: Evaluation of acclimation effects. Eur. J. Agron. (In review).
Lee, M. and R.L. Phillips. 1988. The chromosomal basis of somaclonal variation. Annu. Rev. Plant Physiol. Plant Mol. Biol. 39:413-437.

Li, R. 2007. St. Augustinegrass improvement for freezing tolerance and semi-dwarf growth habit. North Carolina State Univ., PhD Diss. <http:// repository.lib.ncsu.edu/ir/bitstream/1840.16/ 3275/1/etd.pdf>.

Li, R., R. Qu, A.H. Bruneau, and D.P. Livingston. 2010. Selection for freezing tolerance in St. Augustinegrass through somaclonal variation and germplasm evaluation. Plant Breed. 129:417-421.

Maier, F.P., N.S. Lang, and J.D. Fry. 1994. Evaluation of an electrolyte leakage technique to predict St. Augustinegrass freezing tolerance. HortScience 29:316-318.

Milla-Lewis, S.R., J.A. Kimball, T.E. Claure, T.D. Tuong, C. Arellano, and D.P. Livingston, III. 2013. Freezing tolerance and the histology of recovering nodes in St. Augustinegrass. Intl. Turfgrass Soc. J. 12:523-530.

Moseley, D., A. Patton, and J. Trappe. 2010. Winter hardiness of thirty St. Augustinegrass genotypes. Arkansas Turfgrass Report 2010. Ark. Agr. Expt. Stn. Res. Ser. 593:79-84.

Mulkey, S.E., M.C. Zuleta, J.E. Keebler, J.E. Schaff, and S.R. Milla-Lewis. 2013. Development and characterization of simple sequence repeat markers for St. Augustinegrass. Crop Sci. 54(1):401-412.

National Turfgrass Evaluation Program (NTEP). 2012. National turfgrass evaluation program, Beltsville, MD. 22 Aug. 2015. <http://www.ntep.org>.

Philley, H.W., J. Krans, C. Watson, J. Goately, V. Maddox, and M. Tomaso-Peterson. 1996. Turf performance of St. Augustinegrass cultivars in north Mississippi. Miss. Agr. \& For. Expt. St. Bul. 1043.

Philley, H.W., C.E. Watson Jr., J.V. Krans, J.M. Goatley Jr., and F.B. Matta. 1995. Differential thermal analysis of St. Augustinegrass. HortScience 30:1388-1389.

Philley, H.W., C.E. Watson, Jr., V. Krans, J.M. Goatley, V.L. Maddox, and M. Tomaso-Peterson. 1998. Inheritance of cold tolerance in St. Augustinegrass. Crop Sci. 38:451-454.

Reynolds, W.C., R. Li, K. De Silva, A.H. Bruneau, and R. Qu. 2009. Field performance of mutant and somaclonal variation lines of St. Augustinegrass [Stenotaphrum secundatum (Walt.) Kuntze]. Intl. Turfgrass Soc. Res. J. 11:573-582.

Riordan, T.P., V.D. Meier, J.A. Long, and J.T. Gruis. 1980. Registration of Seville St. Augustinegrass. Crop Sci. 20:824-825.

SAS Inst. Inc. 2011. SAS/STAT 9.3 user's guide. SAS Inst., Inc., Cary, NC.

Schwartz, B.M., K.E. Kenworthy, M.C. Engelke, A.D. Genovesi, and K.H. Quesenberry. 2009. Heritability estimates for turfgrass performance and stress response in Zoysia spp. Crop Sci. 49:2113-2118.

Tan, C., Y. Wu, C.M. Taliaferro, G.E. Bell, D.L. Martin, M.W. Smith, and J.Q. Moss. 2015. Selfing and outcrossing fertility in common bermudagrass under open-pollinating conditions examined by SSR markers. Crop Sci. 54(4): 1832-1837. 